\title{
Polyhouse Model for Promoting Agriculture and Its Related Applications
}

\author{
Sudeepthi Govathoti \\ Assistant Professor, Anurag Group of Institutions, Hyderabad, India \\ sudeepthicse@cvsr.ac.in
}

\begin{abstract}
The polyhouse cultivation method provides high growth and improved productivity. To achieve proper crop management it is necessary to collect the correct information to make decisions. In the current work we considered and proposed a framework which could able to collect the information related to polyhouse atmosphere and harvest status and manage the polyhouse. The polyhouse information is collected from different resources by using sampling method. Sampling method contains different strategies that are Simple random, stratified random, Stratified-systematic, Judgmental. Each method identifies the data in different location and collects the data. The collected data to be stored in the mongo database. The database utilize data cleansing, data scraping, data integration and data wrangling process to arrange the data for relevant user. It helps to fix the data in specific manner in the database. Finally, the user can retrieve the data using the Latent Indexing Algorithm to retrieve the data in specific manner. It helps the system to forecast and take steps on situations intended for completely guarded climatic circumstances. So that farmers can access the relevant information using IoT. After the cultivation process the farmers can also marketing their product directly through IoT.
\end{abstract}

Keywords: Polyhouse, Internet of Things, Latent indexing algorithm, Data wrangling

\section{Introduction}

World Wide net pool is an Associate in nursing extension of linguistics net. The linguistics net describes 2 things. That's a combination and sequence of knowledge from varied sources, and it's chiefly targeting the swap of documents. The linguistics net additionally concerns language for recording however the info relates to world objects [1]. Generally, the linguistics net isn't a machine-process in a position. It cannot be traversed by net agents or applications. In linguistics net the knowledge are taken from completely different sources cannot be without delay accessed and even supposing everybody will say something concerning something, only a few folks are literally detected. We tend to solve the matter of linguistics net by doing a little of its basic assumption [2].

IoT comes below the extending current web and providing association between devices is usually means the IoT [3]. IoT based mostly good environments have some distinctive characteristics like it's some prediction capabilities, freshly increased services, remote watching and higher cognitive process capabilities. IT result will assist in connecting each conveyance part, and furnishes the technical tools to attach sensors and different devices to that

Article history:

Received (May 8, 2019), Review Result (July 10, 2019), Accepted (September 18, 2019) 
infrastructure [4]. Network layer connects the device layer to the application layer. The data storage and retrieval are done by using this Cloud management layer. Application layer providing services to the end user and these services also provided to the third parties [5]. Also IoT has some features like security, provisioning, interoperability, latency, massive number of devices, scalability.

Cultivation is crucial thing to people and it is for a food security. Cultivation also helps to human beings to increase the main food grains and also increase the right animals (the rights of animals to live free from human exploitation and abuse) with conformity to surrounding aspects. India has become an economical possible in the world. Nowadays, Polyhouse is popularly used in many countries especially temperature ones such as Japan, Mexico, the northern lands, Korea, France. Growing vegetables in polyhouse research was conducted at May 2007. The main aim of this research was building a suitable polyhouse in hot climate regions. The polyhouse are also giving a good environment so the plants are growing properly and also produced good healthy foods [6]. There are many advantages because the plants developed in a secured surrounding like a polyhouse. Also it produces the yield diseases and the required environments are used to increase productivity [7]. The Polyhouse method is a new technique in cultivation achieved comfort in agricultural India and can be strongly employed for slot areas of agriculture.

Polyhouse farming and still because the different ways of secured atmosphere agriculture have been derived to create a services small climate, that favors the crop production, can be attainable throughout the year or a part of the year pro re nata. The encompassing parameter ordinarily controlled the temperature, commonly giving heat to manage the cold conditions [8]. The condition is most vital factor in polyhouse. But environments condition additionally includes the cooling to scale back excessive temperatures, management light-weight either shading or adding further light, dioxide levels, relative wet, water, plant nutrients and bug management. Processed system is fashionable polyhouse additionally its normal technology with it's a continuing enhancement because the technology advances.

The enormous heats that are prepared by the shinning tubes and that are unacceptable in most instance Fluorescent tubes are useful as the exclusive source of light. Stable case communicate control the physical devices like pump, fan and heater, a thermistor based Stable case communicate is used. One of the digital outputs of the stable case communicate are triggered by the DAQ card and thus control the energizing coil of the contactor [9][10]. Nowadays the recorded lodging and restaurants are using polyhouse to receive best kind of vegetables and fruits from both periodic and aperiodic season to maintain their influence. We can expect large scale of profit from polyhouse farming [11][12].

\section{Related work}

K. Gopala Krishna et.al.[8] Focuses on observance of the environment parameters of hi-tech polyhouse the Wireless detectors Network (WSN). The Wireless Sensor Node are the heart of universal field. Moreover, embedded technology placed the sector of microcontroller is original and most reliable. Therefore, utilizing established innovation, in light-weight of AVR AT mega 8L microcontroller, the Wireless detector Node created and dead for improvement of WSN for polyhouse applications. In like manner, in sight of AN inserted innovation and also the RF module Zigbee an overseas adult male hub is made public concerning extremely encouraging AVR ATmega8L microcontroller and dead for WSN improvement. To upgrade trim yield one has to offer controlled atmosphere to the harvest. 
Tiantian Guo et. al., [10], The Australian greenhouse industry is essentially ruled by minimal effort hydroponic greenhouses for conveyance of water and supplements to plants to grow an assortment of vegetable yields including cucumber and tomato. The water which is the supplement rich drainage water from these greenhouses is for the most part discharged into the nearby environment creating contamination concerns. The study demonstrated that the recycling of the drainage water brought about a 33\% decrease in potable water utilized for water system as a part of cucumber generation. The drainage water contained $59 \%$ connected N, 25\% connected $\mathrm{P}$ and $55 \%$ connected $\mathrm{K}$ and represented the potential for nutrient recuperation and generation cost investment funds through the reuse of drainage water. This contextual analysis shows that some moderately straightforward changes in water system hones inside greenhouse frameworks to reuse drainage water can significantly enhance supportability of ease hydroponic greenhouses and minimize the natural impression of the greenhouse industry.

Kanwal Sachdev et. al., [4], the off-season nursery creation was discovered productive under low-cost polyhouse of 50-meter square zone. Essentially, development of high value off-season vegetables under low cost ensured structures was found a reasonable innovation for developing vegetables effectively amid winter with impermanent insurance from November to February.

\section{Proposed methodology}

In this paper we propose the Poly houses information that are stored in the database and also retrieved the information by relevant users. As shown in [Figure 1], the proposed technique consists of the following blocks. The Poly house information are collected by particular method to be followed as well as the data are being collected. Then the collected information is stored in the multi-dimensional database. The multi-dimensional database using different scheme to stored and provide the user required information. Finally, the information will be retrieved by user using efficient algorithm. The main modules are data collection strategies of poly house information, data collection, Data stored in the mongo database, finally the information retrieval. Below [Figure 1] show that the workflow of the proposed method.

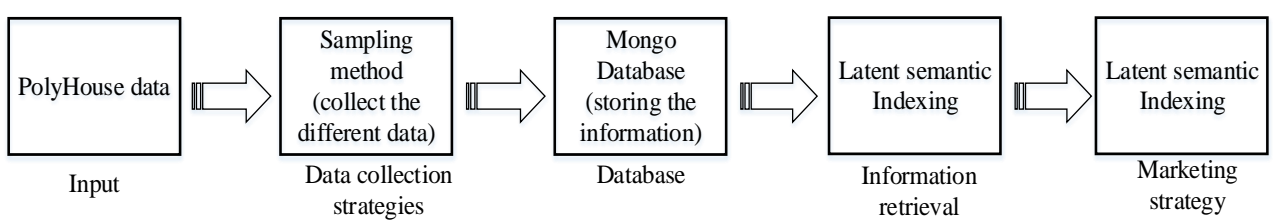

Figure 1. Block diagram for proposed system.

\subsection{Data collection}

The objective for all data collection is to catch quality proof that then means rich data analysis and permits the working of a persuading and credible answer to questions that have been posted. In this proposed method poly house information is collected from various resources. In previous block describe how the data to be collected and that include the properties of the data. The various levels and fields of data to be collected using sampling strategies. Then the collected information is arranged in the data collection block.

In example the data collection for any vegetable that relevant area to be located and vegetable growing temperature, property of the vegetable to be collected. The properties of the data represents the vegetable growing temperature, atmosphere, humidity of the vegetable and the required pesticides of the vegetable, amount of soil using in the plant that to be included. In this each properties of the data to be important for the collecting the data because of the user 
required any kind of information so the data collected in every field with any properties of the plant.

Sample collection involves the data collection strategies and the way of approach in the field. Each data in the field having specific properties and the measuring experiments. Even though the collection of data not to be arranged that viewed in the randomized manner. So the users need data not to be picked in the normal format. So, using different collection strategies for collecting the data in specific manner and then the data to be stored in the database that are explain briefly in the following subsection.

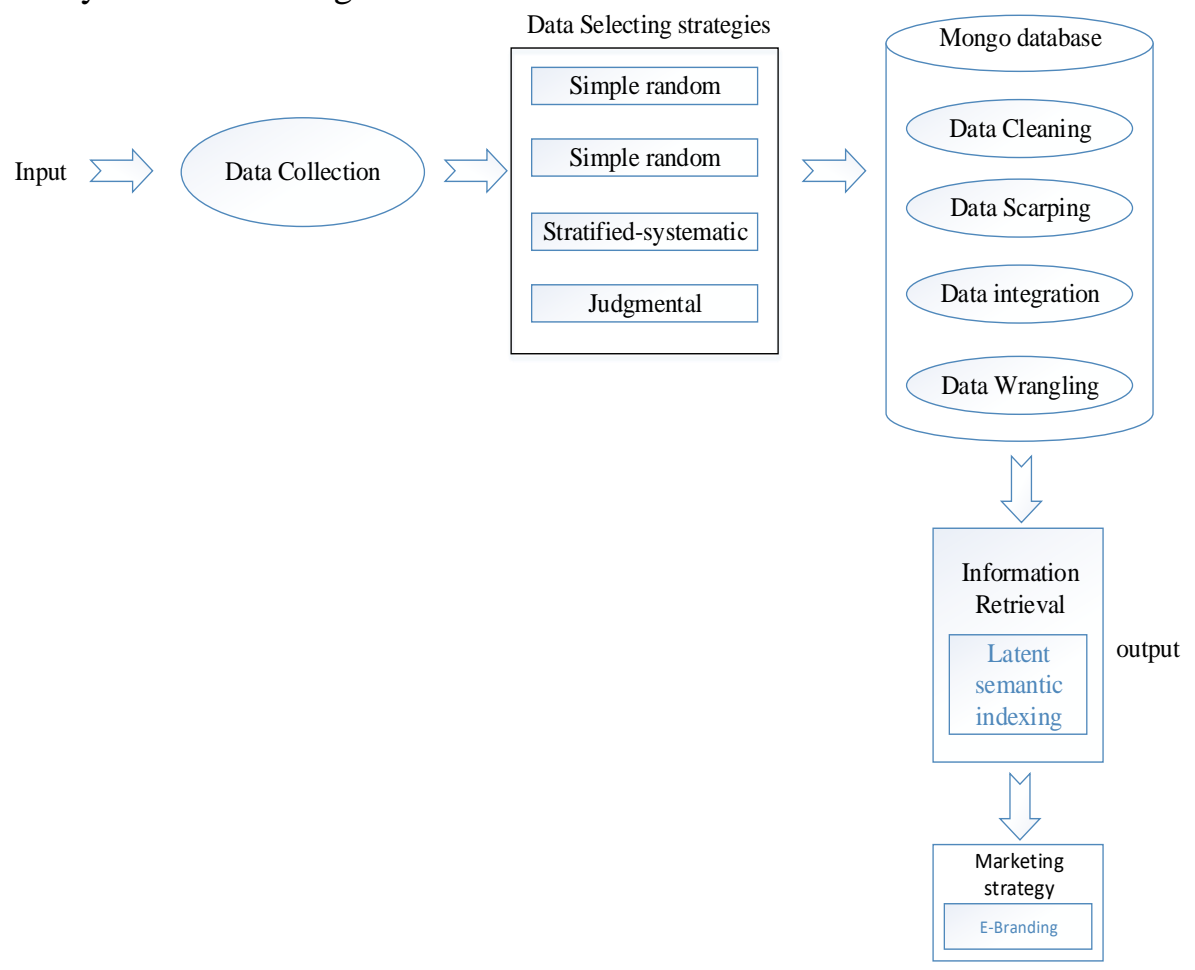

Figure 2. Proposed methodology

\section{Mongo database}

Mongo decibel documents influence to possess all information for a given record during a single document, supposing during an electronic database service information for a given record is sometimes unfold across several tables. During a computer database, the info model would contain multiple tables like classes, Tags, Users, Comments and Articles shown in [Figure 3]. In MongoDB the info can be designed as 2 collections, one for users, and also the different for articles. In every diary document there may be multiple comments, multiple tags, and multiple classes, every expressed as AN embedded array.

Data as documents is simple for developers, quicker for users. In our planned system the classified information may be hold on in Mongolian monetary unit info as rows and columns. However the user would like info are retrieved from the info victimization economical algorithmic rule techniques that in brief delineated as given subsection. 


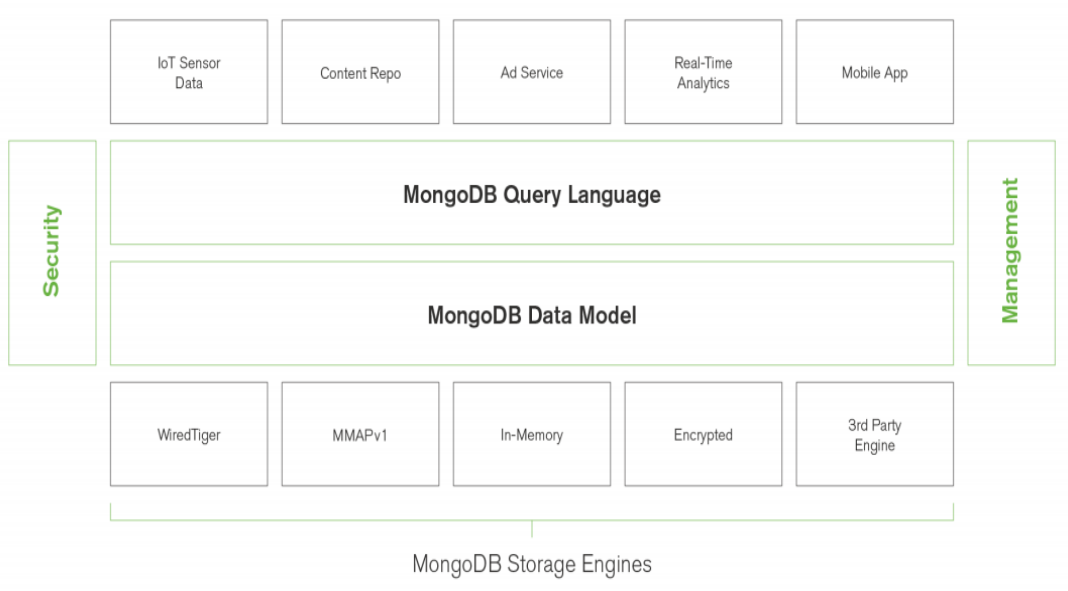

Figure 3. Mongo DB data model

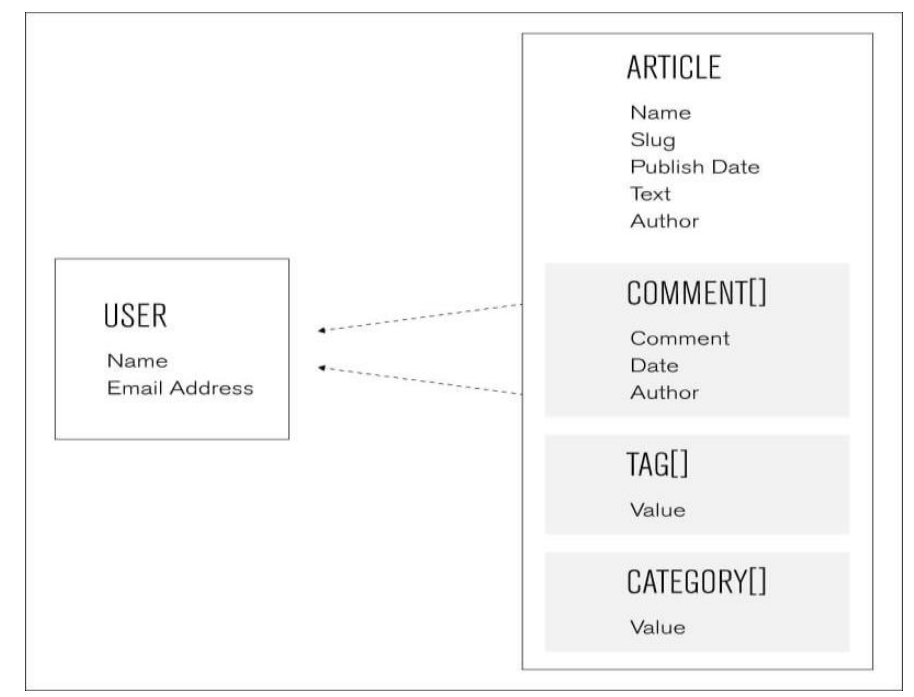

Figure 4. Data as documents

\subsection{Data cleansing}

Data cleansing is that the method of investigation and removing corrupt or inaccurate records from a record set, table, or info. The contradictory information detected or removed by cleansing method which will are elicited by user entry errors, corruption in transmission or storage, totally different information wordbook definitions of comparable entities in several stores. While not utilizing information cleansing strategy within the information base system that happens some problems that are, Lack of quality of the information, Loss of trust, declined the user base, Loss of business support and funding.

\subsection{Data scarping}

Data scraping is additionally referred to as net scarping that used for extracting information from websites. In our planned system the information scarping means that the relevant data solely extracted by the method. Once playing the cleansing method we have a tendency to apply 
the information scarping for data extraction. The scarping method keeps the interchange formats and protocols are generally firmly structured, well-documented, simply verify, and keep unclearness to a minimum. Information scraping usually involves neglecting binary information (usually pictures or multimedia system data), show format, redundant labels, redundant comment and different info that is either moot or compel automatic process.

\subsection{Data integration}

During this process, our planned system has a tendency to victimization information integration for desegregation the information from relevant user. the information scarping wont to extract the relevant data from cleansing method at that time the information are integrated by current field of the information. During this method given the every information set having connected data properties and their demand supported the user would like. Information integration seems with increasing density because the size and also the have to be compelled to share existing information collapse. It's become the main target of user would like the actual product and their properties. Applying the theories provides indications on the expedience and issue of knowledge integration.

\section{E-branding}

E-branding refers to the full of a company's values, proficiency, attitudes, vision, mission, temperament and looks that's flaunted to the audience on-line. The final structure of Estigmatization is shown in fig three.10. Stigmatisation has been characterised because the method of making price through the supply of a desirable and protracted supply and client expertise that may satisfy customers and keep them coming. In the current proposed system, the E-branding used as a marketing strategy for selling the product of the poly house. So the relevant information will be provided as the advertisement of the product in a social media. The branding technique provides the product details, quality, growth information, benefits of the product and the other related information will be provided.

\section{Conclusion}

In E-Branding, the whole leaders sometimes have the monetary strength to forestall competitors, and potential competitor's area unit sometimes disinclined to enter the market if existing brands satisfy customers. Although disapproval has attracted considerable analysis attention, its role and contribution to business performance have remained contentious. Whereas several analysts have foretold the departure of e-brands, others have argued that success on the web is all regarding disapproval. the previous dispute that brands have very little to contribute within the net marketplace and will, indeed, not be continuous because of the extent of worth clarity related to the web medium, the increasing convenience, through a number of easy keystrokes, of subtle search engines and product-comparison tools therefore products/services and willing sellers may be accessed on-line. E-Branding offers straightforward survival of farmer among the competitors. It provides familiarity, loyalty and name to customers.

\section{References}

[1] K. N. Thirukkuralkani, Sandeep Kaushik, and Christy John Ninan, "LabVIEW based greenhouse automation system through wireless protocol," 2nd International Conference on Inventive Systems and Control (ICISC), Coimbatore, pp.1427-1430, (2018) DOI: 10.1109/ICISC.2018.8399082 
[2] Kanwal Sachdeva, Neeraj Kuma, and Rajesh Kumar, "Monitoring and controlling of environmental parameters of polyhouse based on lab VIEW," September, vol.4, no.9, pp.25-32, (2015)

[3] Sidhant S. Kulkarni and S. K. Gupta, “Android application development for polyhouse control,” Global Journal of Engineering Science and Research Management, vol.3, no.5, pp.113-116, (2016)

[4] Yogesh R. Sonawane, Sameer Khandekar, Bipin Kumar Mishra, and K. K. Soundra Pandian, "Environment monitoring and control of a polyhouse farm through internet," World Bank: India Country Overview, vol.11, pp.1-6, (2008).

[5] R.K.Yadav, P.Kalia, H. Choudhary, and Zakir Husainand Brihama Dev, "Low-cost polyhouse technologies for higher income and nutritional security," International Journal of Agriculture and Food Science Technology, vol.5, no.3, pp.191-196, (2014)

[6] Vikas Sharma1 and Neelu Jain, "Polyhouse cultivation using embedded system-A review," International Research Journal of Engineering and Technology (IRJET), vol.3, no.5, pp.1161-1165, (2016)

[7] Khandelwal S.A., "Automated green house management using GSM modem," International Journal of Computer Science and Information Technologies (IJCSIT), vol.3, no.1, pp.3099-3102, (2012)

[8] Gautam I. and Reddy S.R.N., "Innovative GSM bluetooth based remote controlled embedded system for irrigation," International Journal of Computer Applications, vol.47, no.13, pp.1-7, (2012)

[9] Kumar N.S., Krishna B.V., and Agarwal A., "Automatic corporate farming control mechanism (Using embedded systems and GSM technologies)," International journal of Engineering Research \& Management Technology, vol.1, no.1, pp.241-247, (2014)

[10] Pawar S.D. and Rane U.A., "Environment monitoring and device control using ARM based embedded controlled sensor network," SSRG International Journal of Electronics and Communication Engineering (SSRGIJECE), Jan, vol.2, no.1, pp.34-37, (2015)

[11] Sahu K. and Mazumdar S.G., "Digitally greenhouse monitoring and controlling of system based on embedded system," International Journal of Scientific \& Engineering Research (IJSER), vol.3, no.1, pp.1-4, (2012)

[12] Kiran E. Borade, C.S.Patil, and R.R.Karhe, "Polyhouse automation system," International Journal of Advanced Research in Computer Science and Software Engineering, August, vol.3, no.8, pp.602-607, (2013) 
Polyhouse Model for Promoting Agriculture and Its Related Applications

This page is empty by intention. 